Enfermagem Brasil 2018;17(2):162-9

\title{
REVISÃO \\ Influência de gênero em sintomas de síndrome coronariana aguda
}

Josemare Tosta Daiube Santos*, Jonathan Bastos Cruz**

*Graduanda do curso de Enfermagem da Universidade Salvador (UNIFACS), "*Enfermeiro, docente da Universidade Salvador (UNIFACS), especialista em urgência e emergência e UTI adulto

Recebido em 12 de outubro de 2017; aceito em 22 de novembro de 2017.

Endereço para correspondência: Josemare Tosta Daiube Santos, Rua Alameda Carrara, 64, Edf Residencial Carrara, Pituba, 41830-590 Salvador BA, E-mail: josy.tds@gmail.com; Jonathan Bastos Cruz: jonbcruz@gmail.com

\section{Resumo}

A Síndrome Coronariana Aguda (SCA) é um dos achados mais frequentes na prática clínica, que continua a apresentar grande morbidade e mortalidade, apesar dos avanços para seu tratamento. Este estudo tem como objetivo verificar quais são as diferenças na sintomatologia apresentada pelos gêneros na SCA com base em estudos clínicos prévios. Foi realizada uma revisão sistemática de artigos científicos publicados sobre o tema no período de 2006-2016, utilizando-se de bases de dados eletrônicas Lilacs, Capes, Pubmed e OvidMedline, nos idiomas português e inglês. Foram observadas significativas diferenças na apresentação sintomatológica da SCA entre homens e mulheres. Mulheres apresentam mais sintomas atípicos e dor precordial atípica ou ausente com mais frequência do que homens. Esse achado é relevante na prática clínica, fazendo-se necessário adotar medidas de alerta à população de risco, principalmente, e aos profissionais de saúde para que se evitem diagnósticos equivocados e se reduzam atrasos no diagnóstico e tratamento desses pacientes com apresentação sintomatológica atípica.

Palavras-chave: síndrome coronariana aguda, angina instável, infarto do miocárdio, sinais e sintomas, gênero e saúde.

\begin{abstract}
Influence of gender in symptoms of acute coronary syndrome

Acute Coronary Syndrome (ACS) is a very common finding in clinical practice, and although its treatment has advanced, its mortality and morbidity rates are still expressively elevated. The present study's aim is to verify the differences of symptomatology between genders during ACS based on preview clinical studies. A systematic review was conducted including scientific articles published about the theme during the period of 2006-2016, using the databases Lilacs, Capes, Pubmed and OvidMedline, in Portuguese and English. Significant differences were observed in the symptomatic presentation of ACS between men and women. Women presented more atypical symptoms and atypical or absent chest pain more often than men. This finding is relevant in clinical practice, being recommended to adopt measures to alert the risk population mainly, and health professionals to avoid misdiagnosis and to reduce delays in diagnosis and treatment of these patients with atypical symptomatology presentation.
\end{abstract}

Key-words: acute coronary syndrome, unstable angina, myocardial infarction, signs and symptoms, gender and health.

\section{Resumen}

Influencia del género en los síntomas de síndrome coronario agudo

El Síndrome Coronario Agudo (SCA) es uno de los hallazgos más frecuentes en la práctica clínica, que sigue presentando una alta morbilidad y mortalidad a pesar de los avances en el tratamiento. Este estudio tiene como objetivo verificar cuáles son las diferencias en los síntomas presentados por género en SCA con base en estudios clínicos previos. Una revisión sistemática de artículos científicos publicados sobre el tema se llevó a cabo durante el período 2006-2016, utilizando como bases de datos electrónicas Lilacs, Capes, Pubmed and OvidMedline, en los idiomas portugués e inglés. Se observaron significativas diferencias en la presentación sintomatológica de la SCA entre hombres y mujeres. Las mujeres presentaron 
más síntomas atípicos y dolor atípico o falta de dolor en el pecho con más frecuencia que los hombres. Este hallazgo tiene relevancia en la práctica clínica, por lo que es necesario tomar medidas de alerta sobre todo para los que estén bajo riesgo, y para profesionales de la salud para que eviten diagnósticos erróneos y reduzcan los retrasos en el diagnóstico y el tratamiento de estos pacientes con manifestaciones clínicas atípicas.

Palabras-clave: síndrome coronario agudo, angina inestable, infarto del miocardio, signos y síntomas, género y salud.

Introdução

As doenças cardiovasculares possuem atualmente grande impacto no Brasil e no mundo [1], em especial a Síndrome Coronariana Aguda (SCA) que ainda continua a ser um importante fator de morbidade e mortalidade - apesar dos avanços no tratamento, formulação e padronização de guidelines, que orientam como proceder diante dessa situação emergencial -, estimando-se que apenas ela seja a causa de metade de todas as mortes causadas por doenças cardiovasculares [2].

Doenças cardiovasculares representam um terço de uma projeção de 47 trilhões de dólares gastos com doenças não transmissíveis nos próximos 20 anos ao redor do mundo [1]. Os custos estimados para Síndrome Coronariana Aguda no Brasil no ano de 2011 foram de $\mathrm{R} \$ 3,8$ bilhões, incluindo custos diretos (hospitalares) e indiretos (perda de produtividade devido à mortalidade por infarto agudo do miocárdio) [3]

A Síndrome Coronariana Aguda é caracterizada por uma série de manifestações clínicas e laboratoriais de isquemia miocárdica aguda, podendo ser classificada em: Angina Instável (Al), Infarto Agudo do Miocárdio sem supradesnivelamento do segmento ST (IAMSSST) e Infarto Agudo do Miocárdio com supradesnivelamento do segmento ST (IAMCSST) [4].

O IAMCSST é caracterizado por completa obstrução da artéria coronária, causando danos que envolvem toda a espessura do músculo cardíaco, já no IAMSSST ocorre obstrução parcial da artéria coronária e não envolve toda espessura do músculo cardíaco. $\mathrm{Na} \mathrm{Al}, \mathrm{o}$ trombo não obstrui totalmente a artéria e as enzimas cardíacas não se elevam [5].

As recentes pesquisam demonstram certa diferença de morbidade e mortalidade entre homens e mulheres após sofrerem um episódio de SCA, sendo a mortalidade entre mulheres maior na maioria dos casos [6]. Verificou-se também que mulheres levam mais tempo para procurar socorro durante um infarto agudo do miocárdio (IAM) do que homens, parte dessas por não relacionar os sintomas com problema cardíaco emergencial [7].

Dor precordial é o principal sintoma para o reconhecimento da SCA, porém até $35 \%$ dos pacientes podem não apresentar dor no peito durante um episódio de SCA [8]. Principalmente mulheres, idosos e pacientes com diabetes estão em maior risco de um diagnóstico equivocado, ou maior demora em serem diagnosticados, pois reportam dor no peito com menor frequência que homens $[9,10]$, o que é um problema relevante levando-se em consideração que tempo é um fator essencial no tratamento e prevenção de complicações da SCA.

Dada a gravidade, peso e custo das doenças coronárias e a dificuldade de promover maior queda em sua morbimortalidade, faz-se necessário refletir sobre possíveis questões que podem estar impedindo esse progresso. Neste sentido, a significativa diferença de mortalidade no sexo feminino em comparação com o masculino na SCA levou à reflexão acerca desta associação com seus sintomas, culminando na pergunta: Qual a influência do gênero em sintomas de síndrome coronariana aguda?

O objetivo geral deste trabalho, portanto, é verificar se há e quais são as diferenças na sintomatologia apresentada pelos gêneros com diagnóstico ou suspeita de SCA baseando-se em estudos clínicos prévios a respeito do tema, fazendo uma avaliação dos resultados e constatando sua relevância para a prática clínica.

\section{Material e métodos}

Esta revisão sistemática se caracteriza como pesquisa exploratória-analítica, baseada em estudos quantitativos. Todo o processo de produção da revisão sistemática, de pesquisa e seleção de artigos, foi realizado de forma padronizada, seguindo protocolo específico.

A busca na literatura foi realizada através de bases de dados eletrônicos Lilacs, Capes, Pubmed e OvidMedline, utilizando os seguintes termos em inglês e português: Síndrome Coronariana Aguda, sintomas e gênero. Foi obtido um resultado de 1.184 publicações no total. 
Após análise do tema, resumo, exclusão de resultados repetidos, leitura íntegra dos artigos e avaliação metodológica foram selecionados 16 artigos. Foram também revisadas as referências bibliográficas desses artigos.

Os critérios de inclusão para a seleção bibliográfica da presente pesquisa consistiram em estudos publicados nos últimos dez anos (2006-2016), em português e inglês, que continham texto na íntegra e tinham como objetivo principal analisar a apresentação sintomática de pacientes na Síndrome Coronariana Aguda analisando as diferenças entre os gêneros feminino e masculino, sendo esta classificada como IAMCSST, IAMSSST ou angina instável. Foram excluídos estudos que não fizeram comparação e/ou análise de sintomas durante a SCA entre os sexos, que reportaram pacientes com doença arterial coronariana ou que foram publicados há mais de dez anos. Todos os estudos selecionados foram revisados na íntegra pelos autores.

Foram extraídos os seguintes dados dos artigos selecionados: Nome dos autores, localidade do estudo, tipo de estudo, tipo de SCA, método de coleta de dados, período de realização do estudo, tamanho da amostra, idade média dos participantes, ajuste por idade ou outros fatores relacionados e resultado final do estudo (diferenças significativas de sintomas encontrados entre homens e mulheres).

Devido à heterogeneidade dos estudos encontrados não foi realizada metanálise dos dados apresentados, apenas estatística descritiva simples.

Resultados e discussão

Diferenças entre os gêneros na síndrome coronariana aguda: pré-hospitalar, tratamento e mortalidade intrahospitalar

Estudos demonstram que mulheres tendem a demorar mais tempo para buscar atendimento médico de emergência, em comparação com homens, durante um episódio de SCA $[7,11]$. Dentre os fatores envolvidos estão o não reconhecimento dos sintomas como cardíacos e o fato de sua experiência em relação aos sintomas não ser compatível com sua expectativa e idealização do que seria ter um IAM [12]. Estes resultados estão compatíveis com outras pesquisas que referem que mulheres sentem dor precordial em menor proporção que os homens, ou dor menos intensa e apresentam sintomas atípicos com maior frequência na SCA [6].

Além disso, há evidência de que mulheres são menos invasivamente examinadas e tratadas do que homens [11,13-16], tem pior prognóstico e em alguns estudos maior mortalidade intra-hospitalar [11]. Entre as possíveis especulações do motivo pelo qual as mulheres são menos examinadas e tratadas invasivamente, considera-se a apresentação de sintomatologia atípica [16].

\section{Sintomas típicos e atípicos da SCA}

Os sintomas clássicos ou típicos associados à síndrome coronariana aguda são a dor precordial, desconforto no peito, desconforto em outras áreas superiores do corpo, dispneia, suor frio, náusea e tontura, segundo a Associação Americana de Cardiologia [17]. Porém, encontram-se na literatura diversos relatos de sintomatologia sem a clássica dor no peito, ou dor no peito em menor intensidade e mais difusa. Segundo um registro global de eventos coronarianos agudos, cerca de $8 \%$ das pessoas não apresentaram dor precordial ao buscarem atendimento durante um episódio de SCA, e $23,8 \%$ dessas interpretaram os sintomas como sendo de outros tipos de problemas cardíacos ou não cardíacos [18].

Dores atípicas não precordiais são descritas como dor nos ombros, sudorese, palpitações, dor na área superior e média das costas, dispneia, dor nos braços, fadiga excessiva, náusea, vômito, tontura, indigestão, dor na mandíbula, fraqueza e dormência em membros $[6,10]$. Caracterizam-se como dores atípicas principalmente na ausência de dor precordial, pois são sintomas que podem estar relacionados com uma variedade de patologias não necessariamente cardíacas. Esses sintomas descritos foram utilizados para construção de questionários e checklist para avaliar sintomas e suas características em estudos que avaliam a influência de determinados fatores como o sexo, idade e comorbidades na síndrome coronariana aguda, portanto, são sintomas previamente validados como preditivos de SCA [10]. 


\section{Diferenças nos sintomas de síndrome coronariana aguda entre homens e mulheres}

Os estudos indicam que há significativa diferença de sintomatologia entre os gêneros na SCA. As principais características e resultados desses estudos são apresentados no Quadro 1. Foram reportados também outros sintomas incomuns que não haviam sido descritos previamente, como dor nas mãos, anorexia, dor no pescoço, dor na garganta, ansiedade e soluço [19-22].

Os sintomas reportados com maior frequência nas pesquisas, descritos como encontrados significativamente mais vezes em mulheres, ou que foram preditivos, ou ainda com maior probabilidade em mulheres foram náusea [19,21,23-25], vômito [19,20,22,23,26] e dor nos ombros [10,12,21-23]. Em seguida, palpitação [19,23-25], dor nas costas [2-22,25] e dor na mandíbula [20-22,26].

Sintomas reportados com menor frequência, mas ainda significativamente mais encontrados em mulheres do que homens foram dor no pescoço [20-22], dor no peito atípica ou ausente [8,12,27], dispneia [23,26], tontura [19,25], fadiga [19,24], fraqueza [22,28], dor de garganta $[21,22]$ e ansiedade $[19,22]$. Por fim, os sintomas reportados mais frequentes em mulheres por apenas um estudo foram anorexia [19], dor [21] e dormência nas mãos [24].

Alguns sintomas ainda demonstraram resultados conflitantes entre diferentes estudos, como a dor no braço reportada em dois estudos como mais frequente em mulheres do que homens [10,21] e em outro mais frequente em homens do que mulheres [20]. Assim como a sudorese uma vez reportada mais frequente em mulheres [28] e outro em homens [20].

Um estudo conduzido avaliando a sensitividade e especificidade de sintomas de SCA entre os sexos encontrou que mulheres diagnosticadas com SCA eram mais propensas a reportar dor no braço, enquanto homens reportaram mais pressão e dor no peito. Foi observado também que dor no ombro e dor no braço foram preditivos de diagnóstico de SCA apenas para mulheres, e que, dor, pressão e desconforto no peito foram sensitivos, mas não específicos para o diagnóstico de SCA, ou seja, sintomas precordiais estão fortemente associados a indivíduos que se apresentam com SCA, porém, a ausência desses sintomas não necessariamente indica que o indivíduo não apresenta a síndrome [10].

Outros dados importantes encontrados foram que a dor no peito foi ainda o sintoma mais comum entre homens e mulheres em alguns estudos [8,22,24,28,29], demonstrando que apesar de haver evidência que uma parcela da população pode não apresentar dor precordial na SCA, ele é de fato o sintoma mais frequente e mais preditivo de SCA. O problema associado a isso é que a parcela menor da população que não apresenta dor precordial sofre com atrasos no diagnóstico e tratamento, por ter sintomas menos associados à SCA, o que é grave, visto que tempo é um fator essencial na diminuição de morbimortalidade entre os pacientes com essa condição.

Algumas pesquisas mostraram não haver diferença na frequência em que homens e mulheres reportaram dor precordial $[23,25,30]$, enquanto outra encontrou que homens relataram o sintoma mais vezes que mulheres [20]. Poucos estudos relataram ainda não haver diferença significativa de sintomas entre gêneros [30,31], entretanto estes tiveram uma amostra populacional pequena comparado a outras pesquisas que demonstraram diferenças significativas. Devido à inconsistência nos resultados dos estudos, encontram-se divergências de opiniões a respeito de adotar medidas para alertar a população e profissionais de saúde.

Mulheres reportaram maior número de sintomas do que homens em algumas pesquisas, o que está de acordo com resultados encontrados em outros estudos [8,25], demonstrando que como esperado, mulheres tendem a apresentar maior número e variedade de sintomas na SCA do que homens, e sintomas considerados atípicos, principalmente na ausência de dor precordial.

Os resultados deste estudo são compatíveis com os resultados de outras revisões sistemáticas [32,33], em que foram encontradas diferenças substanciais de sintomas entre homens e mulheres no IAM, e dor precordial atípica ou ausente em mulheres em maior proporção que homens. Os resultados de metanálise [33] ainda demonstraram diferenças nos sintomas de modo geral entre os sexos de uma magnitude que garante consideração e significância clínica, sendo necessário alertar o público e os profissionais de saúde quanto a apresentações atípicas de síndrome coronariana aguda, especialmente entre mulheres para redução do tempo de diagnóstico e tratamento e redução da morbimortalidade desses pacientes. 


\section{Características demográficas e clínicas}

Quanto aos dados demográficos e clínicos das pesquisas, no geral, mulheres eram significativamente mais velhas que homens na maioria dos estudos $[14,19,20,23-27,30]$, e tinham maior probabilidade de ter diabetes $[8,21,22,28,30]$ e hipertensão arterial (HAS) $[8,20$ $23,28]$ do que homens, já os homens tinham histórico de tabagismo mais frequentemente que mulheres $[10,19,21,22,30]$. Apenas dois estudos informaram sobre mortalidade, e em ambos as mulheres tiveram maior índice $[21,27]$, dois estudos compararam também o tratamento entre homens e mulheres diagnosticados com SCA e em ambos as mulheres receberam tratamento invasivo e medicamentoso menos frequentemente que homens, dentre eles Intervenção Coronária Percutânea (ICP), cirurgia, terapia tromboembolítica, nitroglicerina endovenosa e heparina $[23,28]$.

\section{Outros problemas associados à diferença de sintomas na SCA}

Apesar de haver evidências em relação à diferença de sintomas entre os gêneros na Síndrome Coronariana Aguda, há uma divergência de opiniões quanto ao que de fato influencia nessas diferenças. Alguns autores associam a diferença de sintomas não ao gênero em si, mas a fatores como idade, comorbidades, principalmente a diabetes mellitus, ou até mesmo 0 tipo de SCA (AI, IAMCSST, IAMSSST).

As pesquisas demonstram que mulheres são cerca de 10 anos mais velhas do que homens quando apresentam SCA[17]. Uma pesquisa realizada detectou as diferenças de sintomas na SCA entre pessoas mais jovens e mais velhas e encontrou que as mais velhas, em sua maioria mulheres, reportaram com menos frequencia dor no peito e mais frequentemente outros sintomas atípicos de SCA [5]. Porém, outros estudos realizados encontraram diferenças de sintomas entre os sexos mesmo em pacientes mais jovens $[8,27]$, um destes encontrou até mesmo que o grupo que menos apresentou dor/desconforto no peito foram mulheres mais jovens, sendo, portanto, inconclusivo a relação da idade com sintomatologia atípica da SCA [27].

Outro fator importante é que em diversas pesquisas, as mulheres além de serem mais velhas, possuíam também maior número de comorbidades $[8,20-23,28,30]$. Alguns autores associam a sintomatologia atípica e a maior morbimortalidade a esse fator, principalmente ao diabetes mellitus (DM). Um estudo realizado comparou sintomas de indivíduos na SCA com e sem diabetes e encontrou que os que possuíam diabetes tinham uma probabilidade significativamente grande de não apresentar dor no peito e de apresentar fadiga incomum em comparação aos não-diabéticos, não encontrou, porém, diferenças significativas entre outros sintomas [34].

O tipo de SCA é também outro fator considerado como principal nos sintomas apresentados, porém resultados quanto a qual tipo de SCA ser mais relacionado com apresentação de sintomas atípicos são inconsistentes nos estudos, enquanto alguns estudos associam o IAMCSST à maior número de sintomas atípicos [35], outros relatam que o IAMSSST demonstrou ter maior número de pessoas sem dor precordial e como forte preditor de apresentação de sintomatologia atípica [36].

Portanto, algumas variáveis são importantes e devem ser levadas em consideração ao se analisar as diferenças de sintomas entre os gêneros, como idade, comorbidades e tipo de SCA, porém, estudos não conseguiram determinar se de fato essas variáveis são o fator principal na influência dos sintomas da SCA.

\section{Conclusão}

Com base nos resultados da pesquisa foi observado que mulheres levam mais tempo para procurar atendimento de emergência durante a SCA do que homens. Dentre os motivos encontrados estão que elas não detectam ou associam seus sintomas com problemas cardíacos emergenciais e que a experiência percebida por elas de ter um IAM foi diferente de suas expectativas do que seria ter um infarto. Esse resultado demonstra a importância de instruir mulheres sobre a possibilidade de apresentação sintomatológica inespecífica, encontrada mais comumente no sexo feminino, e da necessidade de buscar atendimento médico o quanto antes para um melhor prognóstico e prevenção de complicações. 
Foi observado também que as mulheres recebem tratamentos invasivos e medicamentosos com menor frequência que homens e tem pior prognóstico e maior mortalidade. Esses fatores podem ser associados à idade mais avançada da maioria das mulheres em comparação com homens e a terem mais comorbidades ao sofrerem a SCA.

Foram encontradas relevantes diferenças na sintomatologia de homens e mulheres na SCA. Apesar de encontrar variáveis importantes que podem influenciar na natureza desses sintomas, como a idade, comorbidades e tipo de SCA, os estudos realizados até o momento não foram suficientes para determinar a superioridade desses fatores sobre o gênero.

Mulheres apresentaram mais frequentemente sintomas como náusea, vômito, dor nos ombros, palpitação, dor nas costas e na mandíbula, e ainda dor precordial menos frequente do que homens. Esse resultado demonstra porque algumas mulheres têm dificuldade a identificar seus sintomas como sendo de origem cardíaca, pois na ausência de dor no peito eles se tornam inespecíficos, provocando atraso na procura por atendimento médico e no diagnóstico da síndrome.

O presente estudo enfrentou algumas limitações, como a dificuldade para encontrar pesquisas do assunto, por ser pouco abordado no cenário científico e a heterogeneidade das pesquisas selecionadas. Outro problema também foi o tamanho das amostras, em algumas pesquisas bem pequenas, e o método de coleta de dados com questionários sem padronização, com alguns estudos reportando alguns sintomas e outros não, além disso, a análise de prontuário com relação à sintomatologia tende a ser imprecisa ao descrever todos os possíveis sintomas relatados pelos pacientes.

No entanto, as pesquisas incluídas demonstram essa realidade a nível mundial, o que aponta para significância do tema. Não foram encontradas pesquisas do assunto realizadas no Brasil, fazendo-se necessário a realização de estudos no país para avaliar o quadro a nível local.

De qualquer forma, encoraja-se que sejam adotadas medidas para alertar a população de maior risco, assim como aos profissionais de saúde, principalmente médicos e enfermeiros que atuam na triagem desses pacientes com o objetivo de agilizar o diagnóstico e tratamento e reduzir as taxas de mortalidade, comprovadamente mais elevadas nos pacientes que não apresentam a sintomatologia clássica da SCA.

1. Vedanthan R, Seligman B, Fuster V. Global perspective on acute coronary syndrome: a burden on the young and poor. Circ Res 2014;114(12):1959-75.

2. Zhao Z, Winget $M$. Economic burden of illness of acute coronary syndromes: medical and productivity costs. BMC Health Serv Res 2011;11:35.

3. Teich V, Araujo D. Estimativa de custo da síndrome coronariana aguda no Brasil. Rev Bras Cardiol 2011;24(2):85-94.

4. Bassan F, Bassan R. Abordagem da sindrome coronariana aguda. Revista da Sociedade de Cardiologia do Rio Grande do Sul 2006;07:1-7.

5. Hwang SY, Park EH, Shin ES, Jeong MH. Comparison of factors associated with atypical symptoms in younger and older patients with acute coronary syndromes. J Korean Med Sci 2009;24(5):789-94.

6. Canto JG, Goldberg RJ, Hand MM, Bonow RO, Sopko G, Pepine CJ et al. Symptom presentation of women with acute coronary syndromes: myth vs reality. Arch Intern Med 2007;167(22):2405-13.

7. Damasceno CA, Queiroz TL, Santos CAST, Mussi FC. Fatores associados à decisão para procura de serviço de saúde no infarto do miocárdio: diferenças entre gêneros. Rev Esc Enferm USP 2012;46(6):1362-70.

8. Khan NA, Daskalopoulou SS, Karp I, Eisenberg MJ, Pelletier R, Tsadok MA et al. Sex differences in acute coronary syndrome symptom presentation in young patients. JAMA Intern Med 2013;173(20):1863-71.

9. DeVon HA, Penckofer S, Larimer K. The association of diabetes and older age with the absence of chest pain during acute coronary syndromes. West J Nurs Res 2008;30(1):130-144.

10. DeVon HA, Rosenfeld A, Steffen AD, Daya M. Sensitivity, specificity, and sex differences in symptoms reported on the 13-item acute coronary syndrome checklist. J Am Heart Assoc 2014;3(2):e000586. 
11. Leurent G, Garlantezec R, Auffret V, Hacot JP, Coudert I, Fillipi E, et al. Gender differences in presentation, management and inhospital outcome in patients with STsegment elevation myocardial infarction: data from 5000 patients included in the ORBI prospective French regional registry. Arch Cardiovasc Dis 2014;107(5):291-8.

12. King KB, McGuire MA. Symptom presentation and time to seek care in women and men with acute myocardial infarction. Heart Lung 2007;36(4):235-43.

13. Alfredsson J, Stenestrand U, Wallentin L, Swahn E. Gender differences in management and outcome in non-ST-elevation acute coronary syndrome. Heart 2007;93(11):1357-62.

14. Radovanovic D, Erne P, Urban P, Bertel O, Rickli H, Gaspoz JM. Gender differences in management and outcomes in patients with acute coronary syndromes: results on 20,290 patients from the AMIS Plus Registry. Heart 2007;93(11):1369-75.

15. Sielski J, Sadowska AJ, Sadowski M, Kozielska EN, Gierlotka M, Polonski L et al. Differences in presentation, treatment and prognosis in elderly patients with non-STsegment elevation myocardial infarction. Pol Arch Med Wewn 2012;122(6):253-61.

16. Hvelplund A, Galatius S, Madsen M, Rasmussen JN, Rasmussen S, Madsen JK, et al. Women with acute coronary syndrome are less invasively examined and subsequently less treated than men. Eur Heart J 2010;31(6):684-90.

17. Go AS, Mozaffarian D, Roger VL, Benjamin EJ, Berry JD, Borden WB, et al. Heart disease and stroke statistics--2013 update: a report from the American Heart Association. Circulation 2013;127(1):e6-e245.

18. Brieger D, Eagle K, Goodman S, Steg PG, Budaj A, White K, et al. Acute coronary syndromes without chest pain, an underdiagnosed and undertreated high-risk group: insights from the global registry of acute coronary events. Chest 2004;126(2):461-9.

19. Ghezeljeh TN, Tessma MK, Emami A. Sex differences and symptoms of acute coronary syndrome. Client-Centered Nursing Care 2015;1(3):119-24.

20. Arslanian-Engoren C, Patel A, Fang J, Armstrong D, Kline-Rogers E, Duvernoy CS, et al. Symptoms of men and women presenting with acute coronary syndromes. Am J Cardiol 2006;98(9):1177-81.

21. Kosuge M, Kimura K, Ishikawa T, Ebina T, Hibi K, Tsukahara K et al. Differences between men and women in terms of clinical features of st-segment elevation acute myocardial infarction. Circ J 2006;70:222-6.

22. Nia SHS, Haghdost AA, Nazari R, Rezaie R, Sa'atsaz S, Andi SJS, et al. Difference in clinical symptoms of myocardial infarction between men and women. Iranian Journal of Critical Care Nursing 2011;4(1):33-8.

23. Noureddine S, Arevian M, Adra M, Puzantian H. Response to signs and symptoms of acute coronary syndrome: differences between lebanese men and women. American Journal of Critical Care 2008;17(1):26-35.

24. DeVon HA, Ryan C, Ochs A, Shapiro M. Symptoms across the continuum of acute coronary syndromes: differences between women and men. American Journal of Critical Care 2008;17(1):14-24.

25. Berg J, Bjorck L, Dudas K, Lappas G, Rosengren A. Symptoms of a first acute myocardial infarction in women and men. Gend Med 2009;6(3):454-62.

26. Behbahani N, Etemad K, Mehrabi Y, Ahmadi A, Hasani J. Difference in clinical manifestations of myocardial infarction between men and women in Iran in 2014-2015. International Journal of Epidemiologic Research 2016;3(2):106-11.

27. Canto JG, Rogers WJ, Goldberg RJ, Peterson ED, Wenger NK, Vaccarino V, et al. Association of age and sex with myocardial infarction symptom presentation and inhospital mortality. JAMA 2012;307(8):813-22.

28. Omran S, Al-Hassan M. Gender differences in signs and symptoms presentation and treatment of Jordanian myocardial infarction patients. Int J Nurs Pract 2006;12(4):198204.

29. Rosenfeld AG, Knight EP, Steffen A, Burke L, Daya M, DeVon HA. Symptom clusters in patients presenting to the emergency department with possible acute coronary syndrome differ by sex, age, and discharge diagnosis. Heart Lung 2015;44(5):368-75.

30. Bruins Slot MHE, Rutten FH, van der Heijden GJMG, Doevendans PA, Mast EG, Bredero AC, et al. Gender differences in pre-hospital time delay and symptom presentation in patients suspected of acute coronary syndrome in primary care. Fam Pract 2012;29(3):332-37.

31. Pinto $D$, Lunet $N$, Azevedo A. Prevalência e determinantes de apresentação atípica de síndrome coronário agudo. Acta Med Port 2011;24(S2):307-18. 
32. Coventry LL, Finn J, Bremner AP. Sex differences in symptom presentation in acute myocardial infarction: a systematic review and meta-analysis. Heart Lung 2011;40(6):477-91.

33. Shin JY, Martin R, Suls J. Meta-analytic evaluation of gender differences and symptom measurement strategies in acute coronary syndromes. Heart Lung 2010;39(4):283-95.

34. DeVon HA, Penckofer S, Larimer K. The association of diabetes and older age with the absence of chest pain dur-ing acute coronary syndromes. West J Nurs Res 2008;30(1):130-44.

35. Kirchberger I, Meisinger C, Heier M, Kling B, Wende R, Greschik C, et al. Patientreported symptoms in acute myocardial infarction: differences related to ST-segment elevation: the MONICA/KORA Myocardial Infarction Registry. J Intern Med $2011 ; 270(1): 58-64$.

36. Canto AJ, Kiefe Cl, Goldberg RJ, Rogers WJ, Peterson ED, Wenger NK, et al. Differences in symptom presentation and hospital mortality according to type of acute myocardial infarction. Heart J 2012;163(4):572-9. 